\title{
Structure of a single palladium nanoparticle and its dynamics during the hydride phase transformation
}

\section{Ana Suzana ( $\nabla$ anaflaviasuzana@gmail.com )}

Brookhaven National Laboratory https://orcid.org/0000-0001-5736-7506

\section{Longlong Wu}

Brookhaven National Laboratory

\section{Tadesse Assefa}

Brookhaven National Laborstory

\section{Benjamin Williams}

Boston College

\section{Ross Harder}

Argonne National Laboratory

Wonsuk Cha

Argonne National Laboratory https://orcid.org/0000-0001-8888-4258

\section{Chun-Hong Kuo}

Boston College

Chia-Kuang Tsung

Boston College https://orcid.org/0000-0002-9410-565X

\section{lan Robinson}

Brookhaven National Lab

\section{Article}

Keywords: Palladium, hydrogen, predominantly

Posted Date: September 24th, 2020

DOI: https://doi.org/10.21203/rs.3.rs-65592/v1

License: (c) (i) This work is licensed under a Creative Commons Attribution 4.0 International License.

Read Full License

Version of Record: A version of this preprint was published at Communications Chemistry on May 11th, 2021. See the published version at https://doi.org/10.1038/s42004-021-00500-7. 
4 Ana F. Suzana ${ }^{1, *}$, Longlong $\mathrm{Wu}^{1}$, Tadesse A. Assefa ${ }^{1}$, Benjamin P. Williams ${ }^{2}$, Ross $\operatorname{Harder}^{3}$, 5 Wonsuk $\mathrm{Cha}^{3}$, Chun-Hong Kuo ${ }^{4}$, Chia-Kuang Tsung ${ }^{2}$ and Ian K. Robinson ${ }^{1,5, *}$

8 11793, USA

$9 \quad{ }^{2}$ Department of Chemistry, Merkert Chemistry Center, Boston College, Chestnut Hill, MA 02467, USA

$10{ }^{3}$ Advanced Photon Source, Argonne National Laboratory, Lemont, IL 60439, USA

$11{ }^{4}$ Institute of Chemistry, Academia Sinica, Taipei 11529, Taiwan

$12{ }^{5}$ London Centre for Nanotechnology, University College London, London WC1E 6BT, UK

14 Palladium absorbs large volumetric quantities of hydrogen at room temperature and ambient 15 pressure, making the $\mathrm{Pd}-\mathrm{H}$ system a promising candidate for hydrogen storage. Here, we use Bragg 16 coherent diffraction imaging to map the strain associated with defects in three dimensions before 17 and during the hydride phase transformation of an individual octahedral palladium nanoparticle, 18 synthesized by using the seed-mediated approach. The displacement distribution imaging unveils 19 the location of the seed nanoparticle in the final nanocrystal. By comparing our experimental 20 results with a finite-element model, we verify that the seed nanoparticle causes a characteristic 21 displacement distribution of the larger nanocrystal. During the hydrogen exposure, the hydride 22 phase is predominantly formed on one tip of the octahedra, where there is a high number of lower 23 coordinated Pd atoms. Our experimental and theoretical results provide an unambiguous method 24 for future structure optimization of seed-mediated nanoparticles growth and in the design of 25 palladium-based hydrogen storage systems.

\section{Introduction}

28 Hydrogen has the highest chemical energy per unit mass compared with other chemical 29 fuels, three times larger than hydrocarbons fuels ${ }^{1}$, and it is considered a relevant technology, 30 having a great potential to limit environmental impacts. How to safely store hydrogen remains a 31 key goal for the advancement of this new technology. One promising hydrogen storage strategy is 
32 the use of certain metals, which can form hydrides and reversibly absorb large amounts of this gas.

33 Hydrogen can be absorbed by palladium $(\mathrm{Pd})$, and this reaction is characterized by three steps:

34 hydrogen molecular dissociation followed by chemisorption of hydrogen atoms on the Pd surface;

35 next, hydrogen diffusion into the Pd subsurface and, lastly, diffusion into the bulk metallic

36 structure ${ }^{2}$. The Pd-hydrogen system $\left(\mathrm{PdH}_{\mathrm{x}}\right)$ can be described by the formation of two distinct

37 phases, whose existence depends on the hydrogen concentration: the hydrogen-poor solid-state

38 solution called $\alpha$ phase, and the hydrogen-rich hydride, the so-called $\beta$ phase. At room temperature

39 (RT), the maximum hydrogen solubility in the $\alpha$ phase is reached when $x=0.017$, while the single

$40 \beta$ phase exists for $\mathrm{x}>0.6$, and intermediate $\mathrm{x}$ values represent the coexistence of both phases ${ }^{3}$. The

41 formation of $\beta$ phase is accompanied by a lattice expansion of $\sim 3.5 \%$ in the $\operatorname{Pd}$ crystalline

42 structure, caused by the absorption of a high number of hydrogen atoms, forming a new crystalline

43 structure. This $\alpha-\beta$ phase transformation in $\mathrm{Pd}$ nanoparticles is of special scientific interest because

44 it shows fast kinetics under ambient conditions, and is a model system for optimization of the

45 efficiency of hydrogen storage materials.

46 It is well known that nanostructured materials present significant thermodynamic

47 deviations from their bulk counterparts, which is mainly attributed to their larger surface area to

48 volume ratio. It is generally unclear if the observed deviations come directly from the diminutive

49 size effect or from inhomogeneities induced in the sample by its surfaces. The majority of studies

50 on the interstitial dissolution of hydrogen in metals leading to phase transformations have been

51 done on ensemble sample formats ${ }^{4}$, prone to heterogeneity, which results in averaged data,

52 disregarding the influence of the size, shape and, crystallinity on the phase transformation process.

53 Further insight can be obtained by measuring single particles under in situ conditions to identify

54 the role of the nanostructure on the chemical reaction. The phase transformation of the $\mathrm{PdH}_{\mathrm{x}}$

55 system has been extensively studied for bulk samples, but the deviations for nanosized individual

56 nanoparticles are only now starting to be investigated, partly due to the recent development of

57 appropriate techniques, such as electron microscopy ${ }^{5-7}$ and plasmonic spectroscopy ${ }^{8,9}$. However,

58 none of these techniques can characterize both the morphology and 3D displacement distribution

59 at the same time during the $\alpha-\beta$ phase transformation. Here, we report a coherent diffraction

60 experiment that shows this transformation, with picometer resolution of the lattice displacements.

61 A common and efficient approach used for the synthesis of nanoparticles is the so-called

62 seed-mediated growth, which is based on two steps: preparation of the seed and the subsequent 
63 injection of this solution as a precursor for the growth of the larger nanoparticles. This method is

64 based on the reduction of the ionic form of the metal precursor onto the seed surface through the use of a reducing agent and it routinely permits the control of both the size and the shape of the final metal nanoparticles. Capping agents are frequently adopted as they selectively adsorb onto a specific crystal facet of the seed particles and consequently slows down the growth of that facet. The resulting shape of the nanocrystals can be controlled by the nature of the seed and the synthesis conditions, such as reducing/capping agents' concentrations, temperature, etc. However, the research into the detailed mechanism of how the seed is incorporated into the final nanoparticles is still lacking. For example, the induced distortion or strain distribution of the final nanoparticle containing the seed has not been measured yet, to the best of our knowledge.

Bragg coherent diffraction imaging (BCDI) is a lensless technique that works by

74 illuminating a sample with a coherent X-ray probe and recording the coherent diffraction pattern 75 of a single Bragg peak from an individual nanocrystal ${ }^{10,11}$. The missing phase of the diffracted 76 radiation is recovered iteratively using phase retrieval algorithms ${ }^{12}$, going back and forth between 77 the reciprocal and real spaces, until convergence is reached between the measured data and the 78 reconstruction. The amplitude of the resulting 3D complex image is proportional to the electron 79 density of the crystal contributing to the Bragg peak and the phase is proportional to the 80 displacement field of the crystalline structure compared with the equilibrium position of their 81 atoms ${ }^{13}$. The technique can reach nanometric real space resolution, usually $10-20 \mathrm{~nm}^{14}$, while the 82 distortions caused by strain are given with subangstrom sensitivity ${ }^{13}$. The possibility of using 83 reactive environments during the measurement makes BCDI an ideal technique to probe dynamic 84 changes and observe the evolution of defects' distribution. In recent years, in situ BCDI has 85 revealed the dynamics occurring during catalytic processes ${ }^{15-17}$, strain mapping and nucleation 86 dislocation during ionic diffusion in Lithium-ion batteries ${ }^{18-22}$, as well as the defect evolution 87 during the hydriding phase transformation of palladium nanocrystals ${ }^{23-25}$. In ref. 22, Ulvestad et 88 al. investigated the dynamics of cubic Pd nanoparticles during the $\alpha-\beta$ phase transformation using 89 BDCI and the results are consistent with hydrogen uptake in the cube corners, followed by the 90 nucleation of the $\beta$ phase in one corner of the nanocube. Very similar dynamics were also observed 91 for cubic Pd nanoparticles using electron microscopy ${ }^{6,26}$.

92 In this work, we use the seed-mediated growth methodology for producing octahedral Pd 93 nanocrystals, rather than the cubes studied in ref. 22. We utilize BCDI for measuring the 
94 displacement distribution of an individual nanoparticle in three-dimensions under pristine 95 conditions at RT and show that the seed is not only visible, but also plays a crucial role in distorting 96 the final crystalline structure of the nanoparticle. This resulting distortion is consistent with finite 97 element analysis (FEA) calculations. To understand the effect of the nanocrystal shape on the 98 phase transition dynamics, the same nanocrystal is measured under a hydrogen partial pressure of $994,410 \mathrm{~Pa}$ and the hydride phase transformation is directly visualized as a function of time. We 100 captured the partial transformation into the $\beta$ phase and show that the diffusivity of the hydrogen 101 atoms into the Pd crystalline structure is coupled with the coordination number and, possibly, 102 lattice displacement. Our results give structural insights into the reaction states as a function of 103 time at the nanoparticle level, showing that the $\beta$ phase is preferentially formed in one corner of 104 the octahedron, following the "cap model" already predicted by the theoretical study shown in ref. 10524.

\section{Results}

Pd colloidal nanoparticles were synthesized following a seed-mediated growth approach (Methods section). In this process, firstly small cubic Pd seeds are synthesized and then used as a

111 solution and further reduction of this metallic cation by the gradual addition of an ascorbic acid 112 solution. In this case, cetyltrimethylammonium bromide (CTAB) molecules were used as the 113 capping agent, leading to residual strain on the nanocrystal surface. A bright-field transmission 114 electron microscopy (TEM) image of the Pd seed is shown in Supplementary Fig. 1a. One major 115 challenge of BCDI measurements is that nanocrystals can move under the beam due to radiation 116 pressure ${ }^{27}$. In order to address this issue, the $\mathrm{Pd}$ nanoparticles were coated with $\mathrm{TiO}_{2}$ as a fixed 117 support to reduce their mobility under the beam. After synthesis, the nanoparticles in colloidal 118 dispersion were drop-casted onto a flat substrate and coated with crystalline $\mathrm{TiO}_{2}$ using atomic 119 layer deposition (ALD), which permits precise control over the layer thickness of the surrounded 120 material. The $\mathrm{TiO}_{2}$ thickness was maintained around $100 \mathrm{~nm}$, as shown in the scanning electron 121 microscopy (SEM) image in Supplementary Fig. 1b. Faceted octahedral nanocrystals with sizes in 122 the range 200-300 $\mathrm{nm}$ are clearly seen in the SEM image of the as-prepared sample. The 123 boundaries between the metallic nanoparticles and the oxide layer are visible, with the former 124 showing brighter contrast. For the hydrogen chemisorption into the Pd surface to occur, the 
125 hydrogen molecules must diffuse through the $\mathrm{TiO}_{2}$ layer. Our results below show little effect of

126 steric hindrance provoked by the oxide layer. As the hydrogen atoms are small, their diffusion into

127 the Pd crystalline structure is apparently not prevented by the presence of the oxide coating. A

128 detailed description of the synthesis and ALD procedures is given in the Methods section.

Figure 1a shows a SEM image of an individual nanocrystal surrounded by the oxide coating. A schematic with the octahedral geometrical representation of the nanocrystal is shown as the black lines, separating the brighter/darker SEM contrast areas. Palladium nanoparticles with octahedral shape have been chosen due to their crystalline nature, where each crystal is confined by eight $\{111\}$ planes, where four facets meet at each of the six $\{100\}$ vertices of the octahedron.

134 This system is used in this work as a prototype for the $\alpha-\beta$ phase transformation because it is expected to reveal the effect of facets and vertices on the transition dynamics. The BCDI experiment was performed at 34-ID-C beamline at Advanced Photon Source (APS). In brief, the

137 focused coherent X-rays illuminate the $\mathrm{Pd}$ nanoparticles inside a gas environmental cell that contains the nanoparticles. A photon-counting area detector recorded the diffraction patterns while the crystal angle was rocked through the Pd (111) Bragg peak.

Figure 1b shows the 3D Bragg peak for the nanoparticle measured in its pristine state 142 oversampling ratio in the diffraction pattern. Two different views of the reconstruction of the 143 nanocrystal in the pristine state are displayed in Fig. 1c, visualized as an isosurface using the open144 source application Paraview ${ }^{28}$, and it is consistent with a single-crystalline octahedral shape, in 145 accordance with the electron microscopy images. The angles between all the facets were estimated 146 to be $71 \pm 2^{\circ}$. The image phase values were sufficiently strong that unwrapping was required, using 147 the code described in the Methods section, and the original reconstruction with wrapped phases is 148 shown in Supplementary Fig. 2. The black arrow denotes the [111] direction of the momentum 149 transfer vector, $\mathrm{Q}$, which is perpendicular to one of the octahedral facets. The scale of the 3D 150 images is calibrated to represent the relative local displacement field, projected along the Q-vector, 151 where positive values (red-colored) are attributed to atomic displacements along the [111] 152 direction, and negative values (blue-colored), along the opposite direction. The displacement field 153 varies significantly over the nanoparticle surface, indicating the presence of strain that may have 154 been introduced on the nanocrystal during the synthesis and/or ALD procedures. These strong 155 inhomogeneities can be clearly seen in the cross-section views of the lattice displacement maps 
shown in Supplementary Fig. 3. The crystal size is approximately $330 \mathrm{~nm}$ from the top to the 157 bottom corner. The particle volume is $9.2 \times 10^{6} \mathrm{~nm}^{3}$ estimated with Paraview ${ }^{28}$ at an isosurface contour level of $20 \%$. The reconstructed electron density for the nanocrystal measured under these conditions, i.e., RT under nitrogen gas, corresponds to the volume of the metallic phase of

160 palladium. In the first view of the reconstruction shown in Fig. 1c, we observe a concave depression of the surface present on the facet containing the (111) Q vector. This feature and the strong phase reversal associated with it were seen in the images using a wide range of parameters in the reconstruction algorithm, meaning that it is not a reconstruction artifact, but a real feature.

164 The presence of this concave region increases the surface to volume ratio, providing further active sites for chemical reactions ${ }^{29}$.

Figure $2 \mathrm{a}$ and $\mathrm{b}$ show the $3 \mathrm{D}$ reconstruction plotted as a semitransparent isosurface for the nanoparticle in its pristine state. In (a), the plotted displacement was thresholded for negative values $(<-0.36 \AA$, blue-colored). It is noticeable that the portion of the nanocrystal within this negative range is present close to the concave portion, in the direction of the $\mathrm{Q}$ vector and it is homogeneous, having its own crystalline identity. Because it has the expected size and roughly

171 cubic shape, we identify this feature as the crystal-growth seed inside the larger nanocrystal.

172 Interestingly, the Pd seed is not located in the center of the larger nanocrystal, suggesting a growth 173 mechanism different from the conformal epitaxial growth, expected and often observed for seed174 mediated growth. This off-center seeding behavior was already seen by Jin et al. ${ }^{30}$ in the epitaxial 175 growth of copper on Pd cores to produce core-shell nanocubes. They called this mechanism 176 'localized epitaxial growth', and attributed it to the coalescence of different Cu shell domains, that 177 nucleated and grew on different parts of the Pd seed. As a result, most of the Pd seeds were not 178 located in the center of the final nanoparticles. Zeng and coauthors ${ }^{31}$ also reported similar results 179 concerning Pd@Ag core-shell nanoparticles. In this case, the asymmetric growth pattern was attributed to the moderate speed of the injection rate of the metal precursor solution, $\mathrm{AgNO}_{3}$, into 181 the as-synthesized Pd seeds.

182 In Fig. 2b, the displacement shown in (a) and positive displacement values (> $0.78 \AA$, red183 colored) are displayed. It is clear that the highly positive displacement values are concentrated 184 close to the top and bottom portions of the nanocrystal, as best visualized in the cross-section 185 shown in Fig. 2c, taken in the position of the transparent plane with red edges (top-left), along the 186 Q vector direction. To explain this displacement pattern, we suggest that the seed would apply an 
outward force on the nanocrystal, in the sideways direction, as indicated by the black arrows in

188 Fig. 2a, and the nanocrystal would react by rotating its two sides, towards the direction of the Q vector, as shown by the black circular arrows in Fig. 2b. To go further in our investigation, we performed finite element analysis (FEA) calculations using the COMSOL Multiphysics software 191 package ${ }^{32}$. The model nanoparticle consisted of a cube (representing the seed) inserted inside the 192 original octahedral nanocrystal shape, imported from its 3D reconstructed image. Both the crystal 193 and seed were specified as metallic Pd, but different coefficients of thermal expansion (CTE) were 194 assigned to them. Changing the temperature of the simulation allowed the differential distortion pattern to be tuned and visualized. A detailed description of the FEA modeling is presented in the Methods section. Figure $2 \mathrm{~d}$ shows the displacement distribution of the $\mathrm{x}$ component (corresponding to the direction of the Q-vector in our experimental data) as a cross-sectional view of the 3D model Pd nanocrystal. The black line square represents the cube position in our model. It can be seen that positive displacement values are pronounced near the top and bottom edges of the nanocrystal, relative to the center, in agreement with the displacement distribution seen for the nanocrystal measured under pristine conditions. Figure 3 shows the phase line plot over the white dashed lines drawn in the nanocrystal's cross-sections (inset) of the experimental data (solid black line) and the FEA model (solid red line). A similar trend is seen in both experimental and theoretical data, with phase values more pronounced in the extremities of the crystal and smoother in the center region, with a distinct "V"-shaped displacement pattern.

Figure $4 \mathrm{~b}$ and $\mathrm{c}$ show cross-sectional views of the experimental data and FEA model, respectively, taken in the positions shown in Fig. 4a. The overall displacement field resemblance is remarkable, with a strong displacement pattern in the center, propagating in $3 \mathrm{D}$ through the direction of the seed in a cylindrical form. This pattern indicates that the seed acts exerting pressure

211 region seen in the center of the cross-section number 2 shown in Fig. 4c, disagreeing with the blue 212 result of the experiment, is attributed to limitations of using a negative CTE to model the seed as 213 a cube in the FEA. In this case, the cross-section position was taken at the end of the cube, and as 214 the cube shrinks during the modeling process, this portion of the crystal will move forward the 215 positive direction of the $\mathrm{x}$ component. The FEA modeling was also done using an ideal symmetric 216 octahedral shape (Supplementary Fig. 4), using the same approach: a cube representing the seed 217 was introduced inside the octahedron and both were denominated as Pd. After applying a different 
218 CTE for both at a given temperature, the displacement field pattern is very similar to the 219 experimental results. The agreement between the experimental result and FEA calculations validates the capability of BCDI to give displacement distribution with subangstrom sensitivity.

For bimetallic nanoparticles in a core-shell arrangement, a substantial lattice mismatch

222 between the two metals is expected to induce significant strain and possibly defects in the 223 crystalline structure over several atomic layers around the interface. In the case of the seed224 mediated approach used in this work, the seed nanoparticle and the larger nanocrystal are the same 225 material, so perfect epitaxial growth is expected. Even though the lattice-match criterion is met, 226 issues related to the presence of an interfacial layer can interfere with perfect epitaxial growth on 227 the seed surface. In the present work, the seed precursor solution was stabilized by $\mathrm{CTA}^{+} \mathrm{B}^{-}$ 228 bilayers on the surface of Pd cubes. Iodide ions were then added (see the details in the Methods section) and, due to the higher adsorption strength of iodide ions than that of bromide ${ }^{33}, \mathrm{a} \mathrm{CTA}^{+} \mathrm{I}^{-}$ 230 bilayer is expected to be formed on the seed surface. When this solution was directly transferred 231 to the so-called growth solution, the presence of the bilayer can influence the nanocrystal growth 232 from the seed surface. In this BCDI work, we demonstrated that the resulting larger crystal is 233 highly distorted by the presence of the seed. To the extent of our knowledge, this is the first work 234 reporting a three-dimensional image of a mono-metallic seeded nanocrystal, where the seed 235 location can be identified inside the nanoparticle.

236 After the pristine state measurement, the pressure inside the cell was increased in one step 237 using a gas mixture of $4 \%$ of hydrogen in nitrogen, corresponding to an approximate hydrogen 238 partial pressure of 4,410 $\mathrm{Pa}$. Then, the same nanoparticle was measured at RT over time. The 239 reconstructions displayed in Fig. 5 show the evolution of the nanoparticle morphology, after 240 hydrogen exposure. The images in states $I, I I$ and $I I I$ were generated by averaging multiple 241 diffraction scans over different hydrogen exposure times: from 4 to $16 \mathrm{~min}$, from 19 to $28 \mathrm{~min}$, 242 and from 31 to $40 \mathrm{~min}$, respectively. The displacement values shown in Fig. 5 were unwrapped, 243 and the original wrapped phase for states I-III are shown in Supplementary Fig. 5. In the Bragg 244 geometry, the electron density derived from the reconstruction comes from the atomic planes in 245 that particular Bragg orientation. The missing Bragg electron density regions in the reconstructions 246 suggest a transition from the Pd hydrogen-poor $\alpha$ phase to the hydrogen-rich lattice expanded $\beta$ 247 phase, the latter being formed for compositions $\mathrm{PdH}_{\mathrm{x}}, \mathrm{x}>0.017^{34}$. While the lattice constant for 248 palladium in the $\alpha$ phase is $3.89 \AA$, very close to that of the metallic state, it is $4.03 \AA$ for the $\beta$ 
phase which has a different cubic crystal structure with a large number of ordered hydrogen atoms

25026 . Therefore, the two phases will appear in different positions in reciprocal space. The (111) Bragg

251 peak of the $\alpha$ phase was centered on the detector, but the one corresponding to the $\beta$ phase was

252 not seen because it was outside the area detector's angular range. Thus, the disappearance of the 253 electron density, mostly in the top tip region in Fig. 5, starting in state $I$, is interpreted as due to 254 the formation of $\beta$ phase in this region. Smaller gaps due to the hydrogen-rich $\beta$ phase are also 255 seen in the concave region in the center of the (111) facet containing the Q vector and also in the 256 bottom tip of the octahedron, as indicated by the black arrows in Fig. 5.

257 For states $I$ and $I I$ the volume percentage of $\beta$ phase is $2.5 \%$, and for state $I I I$, it is $4.5 \%$, 258 predominantly allocated at the top corner of the octahedron. This indicates that the hydrogen is 259 concentrated in the tip regions where there is a high number of lower coordinated Pd atoms. This 260 result is in accordance with the "cap model" ${ }^{24}$, that proposed a two phase-morphology, with the 261 second phase capping the first and a planar interface between the two phases. The authors 262 theoretically calculated the elastic energy for a $10 \%$ volume fraction of $\beta$ phase distributed both 263 as a spherical-cap and as a core-shell structure. For all hydrogen concentrations, the cap model led 264 to a smaller strain energy. This model is in conformity with the results seen here, where the $\beta$ phase 265 nucleates at one corner of the octahedral nanoparticle. Similar findings were reported for the 266 hydrogenation of octahedral and cubic shaped Pd nanocrystals ${ }^{6,23,26}$. In a series of systematic 267 scanning transmission electron microscopy (STEM) and electron energy loss spectroscopy (EELS) 268 measurements, Sytwu et al. ${ }^{26}$ found similar results for cubic- and octahedral-shaped Pd 269 nanocrystals, with an $\alpha-\beta$ phase interface moving linearly in time, indicating that the reaction is 270 rate-limited by some surface reaction mechanism and not by simple diffusion. The corner initiated 271 nucleation is most likely due to either the high number of lower coordinated Pd atoms in these 272 regions or tensile strain at the corners, resulting from the $\alpha$ phase formation in these areas ${ }^{26}$. In 273 fact, the presence of strain can affect the hydrogen diffusivity. For example, Kimizuka et al. ${ }^{35}$, 274 with an ab initio molecular dynamics model, found that for $\mathrm{H}$ atoms in $\mathrm{Pd}$, a hydrostatic strain of $2752.4 \%$ enhanced the hydrogen lattice diffusion rate by around 20 times that of unstrained Pd at RT. 276 In the $\beta$ phase of palladium hydride, the $H$ atoms occupy two possible interstitial sites in the fcc 277 lattice structure of $\mathrm{Pd}$ : octahedral $(\mathrm{O})$ and tetrahedral $(\mathrm{T})$. The diffusion process at the atomic level 278 occurs by thermally activated jumps of $\mathrm{H}$ atoms between $\mathrm{T}$ and $\mathrm{O}$ sites, being the $\mathrm{O}$-sites more 279 thermodynamically stable ${ }^{35-37}$ and therefore, for the bulk $\beta$ phase, only the $\mathrm{O}$ sites are occupied 
280 by the $\mathrm{H}$ atoms under ambient conditions ${ }^{37,38}$. The difference between the energy for hydrogen in $281 \mathrm{O}$ and $\mathrm{T}$ sites is substantially decreased as strain in increased in the Pd crystalline structure ${ }^{35}$. 282 Consequently, besides the $\mathrm{T}$ sites, the $\mathrm{O}$ sites can be occupied as well, the latter being even more 283 stable than the $\mathrm{O}$ sites for strain values $>1.2 \%$. Quasielastic neutron scattering experiments ${ }^{37}$ 284 revealed that for Pd nanocrystals of $8 \mathrm{~nm}$ diameter, $30 \%$ of the $\mathrm{H}$ atoms are located at the $\mathrm{T}$ sites. 285 Because an increase in the Pd lattice constant was seen in the pristine Pd nanoparticles ${ }^{37}$, lattice 286 distortions are expected to play an important role in the dynamics of the diffusibility of hydrogen. Figure $6 \mathrm{a}$ shows the 3D reconstruction for state $I$, where the transparent plane with red edges is displayed as cross-sections in (b) for states I, II, and III. All the cross-sections were taken at the same position. The displacement field distributions are significantly modified from the pristine state measurement (Fig. 2c), mostly in the vicinity of the top and bottom nanocrystal tips. This is very clear in the phase plot line shown in Fig. 6c, which represents the phase distribution along the white dashed lines drawn in (b). A remarkable feature of this plot line is that the phase values at the edges of the particle tend to reverse direction progressively with hydrogen exposure, as indicated by the black arrows in the plot. There is a clear ramping behavior in the phase with abrupt reversals. In BCDI, phase ramps are a direct indication of regions of a crystal with a slightly different lattice parameter, so we can describe the overall distribution seen as blocks of $\alpha$ phase going forward from the top and bottom terminations of the nanocrystal, while the rest of the nanoparticle remains as metallic Pd. This result is in agreement with the preferential hydrogen uptake from the corners of the octahedron, as discussed above.

In this study, we have measured in situ the 3D displacement field distribution in a single octahedral Pd nanoparticle under pristine conditions and during the $\alpha-\beta$ phase transformation induced by hydrogen exposure. The reconstruction revealed the presence of a concave portion on one of the 111 facets, and the region right below it presents a homogeneous displacement distribution that is identified as the seed precursor used in the nanoparticles synthesis. We found that the presence of the seed has an influence on the 3D displacement distribution throughout the volume of the pristine nanocrystal. This characteristic displacement field is fully explained by 307 finite element calculations, modeled with a cube inserted into an octahedral Pd nanoparticle 308 causing rotations of the outer regions. During the hydriding phase transformation, we were able to 309 capture the reaction over time. The reaction stages (states I-III) demonstrate that the hydrogen-rich $\beta$ phase is predominantly formed on one tip of the nanoparticle, where there is a high number of 
311 undercoordinated Pd atoms, and pronounced displaced atoms. Our results show an unambiguous 312 methodology to study the dynamics of phase transformations and provide important insights into 313 the investigation of dynamic processes in individual nanocrystals under realistic conditions. 314 Hydrogen solubility and diffusivity are properties determining how much and how fast the 315 hydrogen will be delivered in the structure and, in this work, we have shown that both properties 316 are highly influenced by the lower coordination number in the vertices rather than surface faceting. 


\section{Methods}

318 Palladium nanocrystals synthesis. The octahedral palladium nanoparticles were synthesized 319 following a seed-mediated growth approach based on a previous report ${ }^{39}$. Cube-shaped palladium 320 seed nanoparticles were synthesized by mixing $0.500 \mathrm{~g}$ of cetyltrimethylammonium bromide 321 (CTAB), $0.5 \mathrm{~mL}$ of a $0.01 \mathrm{M}$ tetrachloropalladinic acid $\left(\mathrm{H}_{2} \mathrm{PdCl}_{4}\right)$ solution, $0.3 \mathrm{~mL}$ of a $0.01 \mathrm{M}$ 322 sodium iodide $(\mathrm{NaI})$ solution and $10 \mathrm{~mL}$ of nanopure water. The resulting solution was maintained 323 in an oil bath for 5 minutes at $95{ }^{\circ} \mathrm{C}$. Then, $200 \mu \mathrm{L}$ of a $0.04 \mathrm{M}$ ascorbic acid solution was added 324 and the temperature was kept at $95{ }^{\circ} \mathrm{C}$ for $1 \mathrm{~h}$. The growth procedure was done by adding together $3250.360 \mathrm{~g}$ of CTAB, $0.250 \mathrm{~mL}$ of a $0.01 \mathrm{M} \mathrm{H}_{2} \mathrm{PdCl}_{4}$ solution, $0.050 \mathrm{~mL}$ of a $0.001 \mathrm{M} \mathrm{NaI}$ solution, 326 and $9.375 \mathrm{~mL}$ of nanopure water. This solution was maintained at $30^{\circ} \mathrm{C}$. After 5 minutes, $80 \mu \mathrm{L}$ 327 of the as-prepared Pd seeds and $250 \mathrm{uL}$ of a $0.04 \mathrm{M}$ ascorbic acid solution were added and the 328 system was maintained at $30{ }^{\circ} \mathrm{C}$ for $40 \mathrm{~h}$.

$\mathrm{TiO}_{2}$ atomic layer deposition. The crystalline $\mathrm{TiO}_{2}$ coating was deposited in a Cambridge nanotech (Savannah 100) system based on a previous report ${ }^{40}$. The reaction was performed at 275 ${ }^{\circ} \mathrm{C}$ with a constant flow of $\mathrm{N}_{2}$ at $20 \mathrm{sccm}$ (base pressure $\sim 100 \mathrm{mTorr}$ ). Titanium isopropoxide ( $\left.\mathrm{Ti}(\mathrm{iPrO})_{4}\right)$ (heated to $75^{\circ} \mathrm{C}$ ) served as the Ti precursor and nanopure $\mathrm{H}_{2} \mathrm{O}$ at room temperature was used as the oxygen precursor. The pulse and purge time for $\operatorname{Ti}(\mathrm{iPrO})_{4}$ and $\mathrm{H}_{2} \mathrm{O}$ were $0.1 \mathrm{~s}$ \& $3355 \mathrm{~s}$ and $0.01 \mathrm{~s} \& 10 \mathrm{~s}$, respectively.

Transmission electron microscopy (TEM) and Scanning electron microscopy (SEM). For the electron microscopy imaging of the seed nanoparticles, $5 \mu \mathrm{L}$ of a concentrated seed solution was drop-casted onto a carbon-coated copper grids and left undisturbed for drying at room temperature. TEM bright-field images were acquired by a JEOL JEM-2100F microscope operating at $200 \mathrm{kV}$.

341 The $\mathrm{TiO}_{2}$ coated Pd nanoparticles were imaged using a Hitachi FlexSEM 1000, operating in an 342 accelerating voltage of $15 \mathrm{kV}$. In this case, the sample was prepared by drop-casting $10 \mu \mathrm{L}$ of the 343 colloidal dispersion on $\mathrm{Si}$ wafer, which was left to dry at room temperature prior to analysis.

345 Bragg coherent diffraction imaging experiment details. The experiment was performed at 34346 ID-C at Advanced Photon Source (APS), Argonne National Laboratory, USA. A double crystal 347 monochromator selected the energy to $9 \mathrm{keV}$ and Kirkpatrick-Baez (KB) mirrors were used to 
348 focus the beam to $600 \times 600 \mathrm{~nm}^{2}$. The coherent diffraction patterns for the Pd (111) Bragg 349 reflection were recorded in a Medipix detector $\left(512 \times 512\right.$ pixels, $\left.55 \times 55 \mu^{2}\right)$, which was placed $3500.5 \mathrm{~m}$ away from the sample. The nanocrystals were drop-casted on a silicon wafer substrate, which 351 was placed inside an in situ cell. The rocking curve of the Pd Bragg peak was collected by using 352 angular steps of $0.02^{\circ}$ in 60 frames, and $2 \mathrm{~s}$ of exposure time. The patterns were recorded 353 continuously during the hydrogen exposure. The $\operatorname{Pd} \alpha-\beta$ phase transformation was induced by 354 flowing a gas mixture composed of $4 \%$ of hydrogen gas in $\mathrm{N}_{2}$ in an approximate partial pressure 355 of $\mathrm{H}_{2}$ of $4,410 \mathrm{~Pa}$.

357 Data reconstruction parameters. Error reduction (ER) and hybrid-input-output (HIO) algorithms 358 were used for the reconstructions, in a total of 620 iterations ${ }^{12,41}$. The phase retrieval code was 359 initiated with 20 ER iterations, alternating with 180 iterations of the HIO algorithm, starting from 360 random phases. A gaussian shrink-wrap function with a threshold of 0.15 was used as the support 361 constraint $^{42}$. The reconstructions were plotted as isosurface contour-maps of the image amplitude 362 in 3D and 2D cross-sections with Paraview ${ }^{28}$. The maps were colored with the image phase, which 363 represents the projection of the local displacement onto the Q-vector.

3D phase unwrapping method. The 3D phase unwrapping algorithm is performed based on a non-continuous path to complete the unwrapping process ${ }^{43}$. The algorithm is used to solve the $2 \pi$ phase discontinuity of the phase extracted from complex volumetric data. For the algorithm, it firstly calculates the reliability of each voxel using the second difference method. Then, with the obtained horizontal, vertical, and normal edges' reliability, the algorithm will sort all of these edges according to their reliabilities. Finally, the algorithm will unwrap voxels according to the edges reliabilities by offsetting these voxels with a multiple of $2 \pi$ to make the input phase change 372 continually.

374 Theoretical approach modelling. Finite element analysis (FEA) was done using COMSOL 375 Multiphysics software ${ }^{32}$. The FEA simulations accounted for materials properties including 376 domain temperature, thermal expansion and three-dimensional object shape. A cube with $50 \mathrm{~nm}$ 377 edge dimensions was united together with the octahedral original shape, imported from the BCDI 378 reconstruction, and both were denominated as metallic Pd. In order to simulate the displacement 
379 field distribution caused by the presence of the seed, the cube was given a different anisotropic 380 coefficient of thermal expansion (CTE), set to $\alpha_{x x}=0, \alpha_{y y}=\alpha_{z z}=-4 \times 10^{-4} \mathrm{~K}^{-1}$ instead of the original 381 CTE for metallic Pd, which is $1.18 \times 10^{-5} \mathrm{~K}^{-1}$. The CTE value was set to 0 for the octrahedral 382 domain, and the temperature was raised by $100 \mathrm{~K}$ in both domains. A second FEA approach was 383 to build an ideal symmetric octahedron plus the cubic shaped object in AutoCAD, that was load in 384 COMSOL Multiphysics, and then the model was built similarly as the one using the original data, 385 except that the CTE $\left(-1 \times 10^{-4} \mathrm{~K}^{-1}\right.$ in this case), was defined isotropically.

387 Data availability. The data discussed in this paper are available upon request to the corresponding 388 authors. Correspondence and requests for materials should be addressed to A.F.S. (e-mail 389 asuzana@bnl.gov) and I.K.R. (e-mail: irobinson@bnl.gov).

Code availability. The code used for the 3D phase unwrapping is available upon request to L.W. 392 (lwu@bnl.gov).

\section{References}

395 1. Schlapbach, L. \& Züttel, A. Hydrogen-storage Materials for Mobile Applications. Nature $396 \quad 353-358(2001)$.

397 2. Jerkiewicz, G., Borodzinski, J. J., Chrzanowski, W. \& Conway, B. E. Examination of 398 Factors Influencing Promotion of H Absorption into Metals by Site-Blocking Elements. $J$. $399 \quad$ Electrochem. Soc. 142, 3755 (1995).

400 3. Manchester, F. D., San-Martin, A. \& Pitre, J. M. The H-Pd (hydrogen-palladium) System. $401 \quad J$. Phase Equilibria 15, 62-83 (1994).

402 4. Bardhan, R. et al. Uncovering the intrinsic size dependence of hydriding phase 403 transformations in nanocrystals. Nat. Mater. 12, 905-912 (2013).

404 5. Narayan, T. C., Baldi, A., Koh, A. L., Sinclair, R. \& Dionne, J. A. Reconstructing solute405 induced phase transformations within individual nanocrystals. Nat. Mater. 15, 768-774 $406 \quad$ (2016).

407 6. Narayan, T. C. et al. Direct visualization of hydrogen absorption dynamics in individual 408 palladium nanoparticles. Nat. Commun. 8, 1-8 (2017).

409 7. Baldi, A., Narayan, T. C., Koh, A. L. \& Dionne, J. A. In situ detection of hydrogen- 
induced phase transitions in individual palladium nanocrystals. Nat. Mater. 13, 1143-1148 (2014).

412 8. Shegai, T. \& Langhammer, C. Hydride formation in single palladium and magnesium nanoparticles studied by nanoplasmonic dark-field scattering spectroscopy. Adv. Mater. 23, 4409-4414 (2011).

415 9. Syrenova, S. et al. Hydride formation thermodynamics and hysteresis in individual Pd nanocrystals with different size and shape. Nat. Mater. 14, 1236-1244 (2015).

10. Pfeifer, M. A., Williams, G. J., Vartanyants, I. A., Harder, R. \& Robinson, I. K. Threedimensional mapping of a deformation field inside a nanocrystal. 442, 63-66 (2006).

11. Clark, J. N., Huang, X., Harder, R. \& Robinson, I. K. High-resolution three-dimensional partially coherent diffraction imaging. Nat. Commun. 3, 993 (2012).

422 13. Robinson, I. \& Harder, R. Coherent X-ray diffraction imaging of strain at the nanoscale. $423 \quad$ Nat. Mater. 8, 291-298 (2009).

424 14. Estandarte, A. K. C. et al. Bragg coherent diffraction imaging of iron diffusion into gold 425 nanocrystals. New J. Phys. 20 (2018).

426 15. Suzana, A. F. et al. In situ three-dimensional imaging of strain in gold nanocrystals during catalytic oxidation. Nanoscale Adv. 1, 3009-3014 (2019).

428 16. Ulvestad, A. et al. In Situ 3D Imaging of Catalysis Induced Strain in Gold Nanoparticles. 429 J. Phys. Chem. Lett. 7, 3008-3013 (2016).

430 17. Kim, D. et al. Active site localization of methane oxidation on Pt nanocrystals. Nat.

431 Commun. 9, 1-7 (2018).

432 18. Ulvestad, A. et al. Topological defect dynamics in operando battery nanoparticles. Science $433 \quad 348,1344-1347(2015)$.

434 19. Singer, A. et al. Nucleation of dislocations and their dynamics in layered oxide cathode 435 materials during battery charging. Nat. Energy 3, 641-647 (2018).

436 20. Singer, A. et al. Nonequilibrium structural dynamics of nanoparticles in $\mathrm{LiNi}_{1 / 2} \mathrm{Mn}_{3 / 2} \mathrm{O}_{4}$ 437 cathode under operando conditions. Nano Lett. 14, 5295-5300 (2014).

438 21. Ulvestad, A. et al. Single particle nanomechanics in operando batteries via lensless strain 439 mapping. Nano Lett. 14, 5123-5127 (2014).

440 22. Ulvestad, A. et al. Nanoscale strain mapping in battery nanostructures. Appl. Phys. Lett. 
104, (2014).

442 23. Ulvestad, A. et al. Avalanching strain dynamics during the hydriding phase transformation in individual palladium nanoparticles. Nat. Commun. 6, (2015).

444 24. Ulvestad, A. et al. Three-dimensional imaging of dislocation dynamics during the hydriding phase transformation. Nat. Mater. 16, 565-571 (2017).

25. Ulvestad, A. \& Yau, A. The self-healing of defects induced by the hydriding phase transformation in palladium nanoparticles. Nat. Commun. 8, 1-6 (2017).

26. Sytwu, K. et al. Visualizing Facet-Dependent Hydrogenation Dynamics in Individual Palladium Nanoparticles. Nano Lett. 18, 5357-5363 (2018).

27. Liang, M., Harder, R. \& Robinson, I. Radiation-driven rotational motion of nanoparticles. J. Synchrotron Radiat. 25, 757-762 (2018).

28. Ahrens, J., Geveci, B., Law, C.: ParaView: an end-user tool for large data visualization. Elsevier, Visualization Handbook (2005)

29. George, C. et al. A cast-mold approach to iron oxide and Pt/iron oxide nanocontainers and nanoparticles with a reactive concave surface. J. Am. Chem. Soc. 133, 2205-2217 (2011).

31. Zeng, J. et al. Controlling the nucleation and growth of silver on palladium nanocubes by manipulating the reaction kinetics. Angew. Chemie - Int. Ed. 51, 2354-2358 (2012).

32. COMSOL Multiphysics ${ }^{\circledR}$ v. 5.5. www.comsol.com COMSOL AB, Stockholm, Sweden.

33. Niu, W., Zhang, L. \& Xu, G. Shape-controlled synthesis of single-crystalline palladium nanocrystals. ACS Nano 4, 1987-1996 (2010).

34. Switendick, A. Hydrogen in Metals I. Springer 101-129 (1978).

35. Kimizuka, H., Ogata, S. \& Shiga, M. Mechanism of fast lattice diffusion of hydrogen in palladium: Interplay of quantum fluctuations and lattice strain. Phys. Rev. B 97, 1-11 (2018).

36. Kofu, M. et al. Hydrogen diffusion in bulk and nanocrystalline palladium: A quasielastic neutron scattering study. Phys. Rev. B 94, 1-11 (2016).

37. Akiba, H. et al. Nanometer-Size Effect on Hydrogen Sites in Palladium Lattice. J. Am. Chem. Soc. 138, 10238-10243 (2016). 
472 38. Worsham, J. E., Wilkinson, M. K. \& Shull, C. G. Neutron-diffraction observations on the palladium-hydrogen and palladium-deuterium systems. J. Phys. Chem. Solids 3, 303-310 (1957).

39. Sneed, B. T., Kuo, C. H., Brodsky, C. N. \& Tsung, C. K. Iodide-mediated control of rhodium epitaxial growth on well-defined noble metal nanocrystals: Synthesis,

40. Lin, Y., Zhou, S., Liu, X., Sheehan, S. \& Wang, D. TiO $2 / \mathrm{TiSi}_{2}$ heterostructures for highefficiencyphotoelectrochemical $\mathrm{H}_{2} \mathrm{O}$ splitting. J. Am. Chem. Soc. 131, 2772-2773 (2009).

41. Gerchberg, R. W. \& Saxton, W. O. A practical algorithm for the determination of phase from image and diffraction plane pictures. Optik (Stuttg). 35, 237-246 (1972).

42. Marchesini, S.; He, H.; Chapman, H.; Hau-Riege, S.; Noy, A.; Howells, M.; Weierstall, U. \& Spence, J. X-ray image reconstruction from a diffraction pattern alone. Phys. Rev. B 68, (2003).

43. Abdul-Rahman, H., Gdeisat, M., Burton, D. \& Lalor, M. Fast three-dimensional phaseunwrapping algorithm based on sorting by reliability following a non-continuous path. Opt. Meas. Syst. Ind. Insp. IV 5856, 32 (2005).

Acknowledgements: A.F.S. and I.K.R. would like to thank Xiaojing Huang for the help with the electron microscopy. B.P.W. and C.-K.T. would like to thank the support from Boston College. Thank you to Connor C. Starkey for assistance with Pd nanoparticle synthesis and to Haochuan Zhang and Dunwei Wang for assistance with ALD coating. This research used resources of Brookhaven National Laboratory, supported by the U.S. Department of Energy (DOE), Office of Basic Energy Sciences, Division of Materials Sciences and Engineering, under Contract No. DESC0012704. The BDCI experiment took place at the Advanced Photon Source, a U.S. DOE Office of Science User Facility operated for the DOE Office of Science by Argonne National Laboratory

Author contributions: A.F.S., L.W., T.A.A., R.H., W.C., and I.K.R. performed the experiment. 501 C.-K.T. and B.P.W. synthesized the sample. L.W. wrote the code for the phase unwrapping 502 procedure. A.F.S. and I.K.R. conducted the finite-element analysis modelling. All the authors 
503 interpreted the results. A.F.S. and I.K.R. wrote the paper and all authors contributed to editing the 504 manuscript.

505

506 Competing financial interest: the authors declare no competing financial interest. 

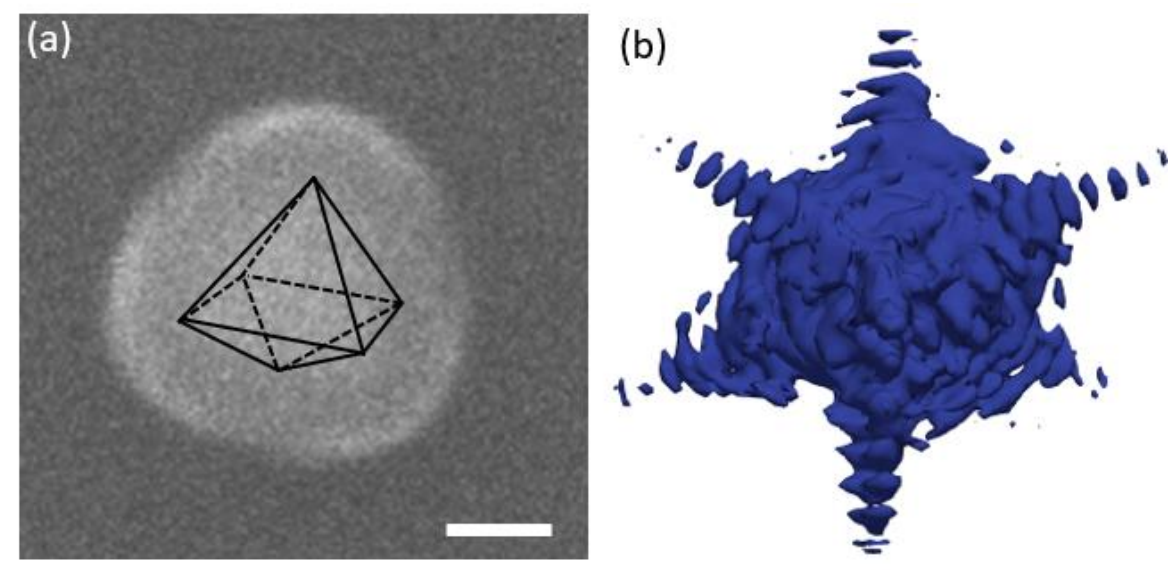

\section{(c)} (111)

\section{0}

511 Figure 1. Imaging the displacement field of the octahedral Pd nanoparticle. (a) Scanning

512 electron microscopy image of an individual $\mathrm{Pd}$ nanoparticle, showing the $\mathrm{TiO}_{2}$ layer surrounding

513 the metallic core. The black dashed lines are a guide for the eyes, distinguishing the Pd surface

514 and the titanium dioxide. (b) Isosurface of the $3 \mathrm{D}$ diffraction pattern obtained from the $\mathrm{Pd}$

515 nanoparticle measured under the pristine state $\left(p_{H_{2}}=0\right)$. (c) Different views of the reconstructed

516 displacement field for the pristine state nanoparticle shown as an isosurface, colored according to

517 its displacement component along the [111] direction, $u_{111}$. The black arrow represents the [111]

518 direction of the measurement Q-vector. The scale bars in (a) and (c) represent $100 \mathrm{~nm}$. 

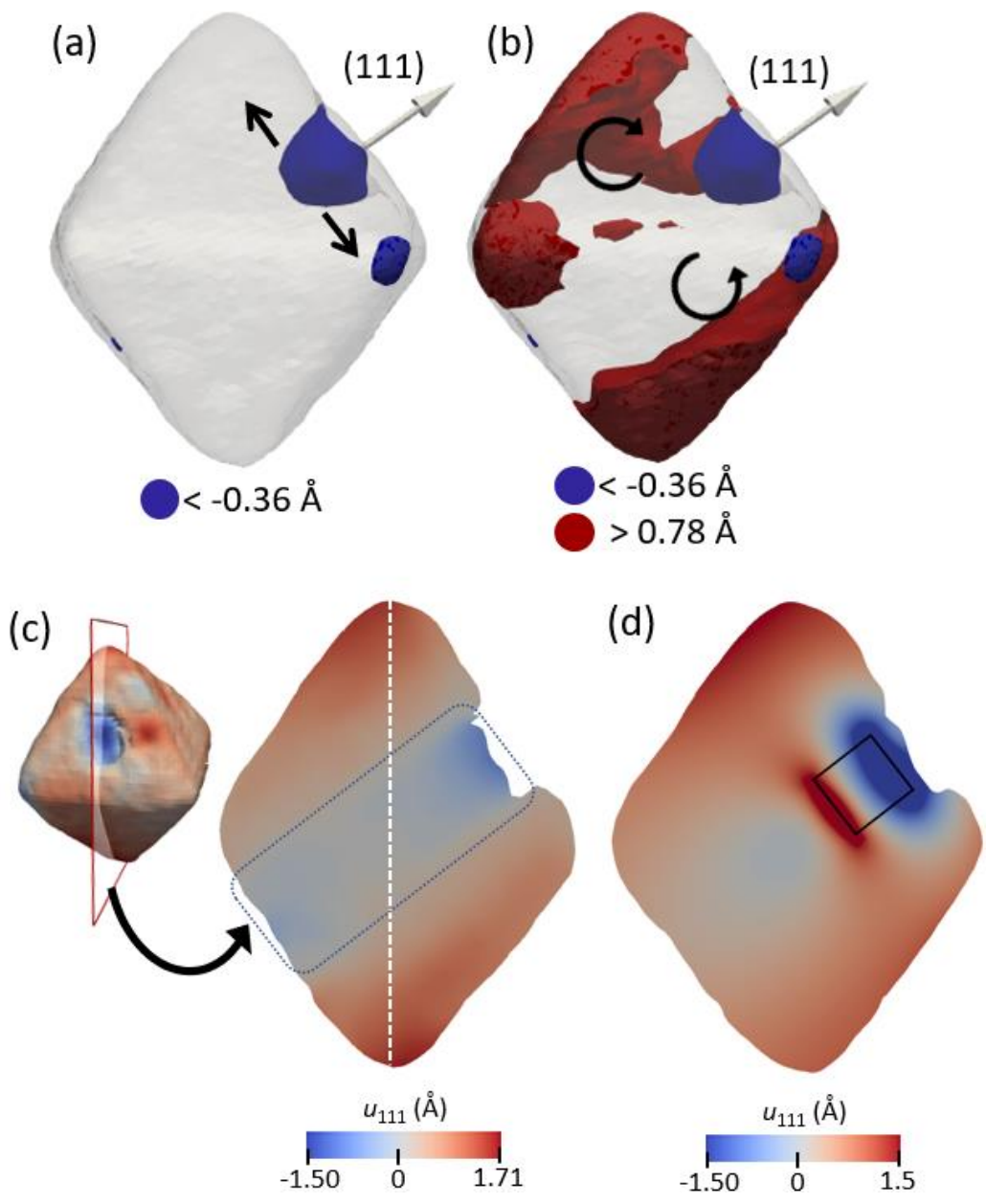

521 Figure 2. Displacement field distribution over the Pd nanocrystal for the measument done

522 under pristine conditions. (a) Semitransparent 3D plot of the Pd nanoparticle showing negative

523 displacement values (<-0.36 A, blue-colored) and (b) the same negative values shown in (a) and 524 positive displacement values (> $0.78 \AA$, red-colored). (c) Cross-section view of the internal 525 displacement field distribution taken along the (111) Q vector. The position of the plane taken 526 from the 3D reconstruction is shown in the top-left. (d) Cross-sectional view of the displacement 527 distribution from the finite element analysis, modeled using the original reconstructed octahedral 528 nanoparticle shape with a cube placed inside it to represent the seed nanoparticle. The square inside 529 the plot represents the cube position. 


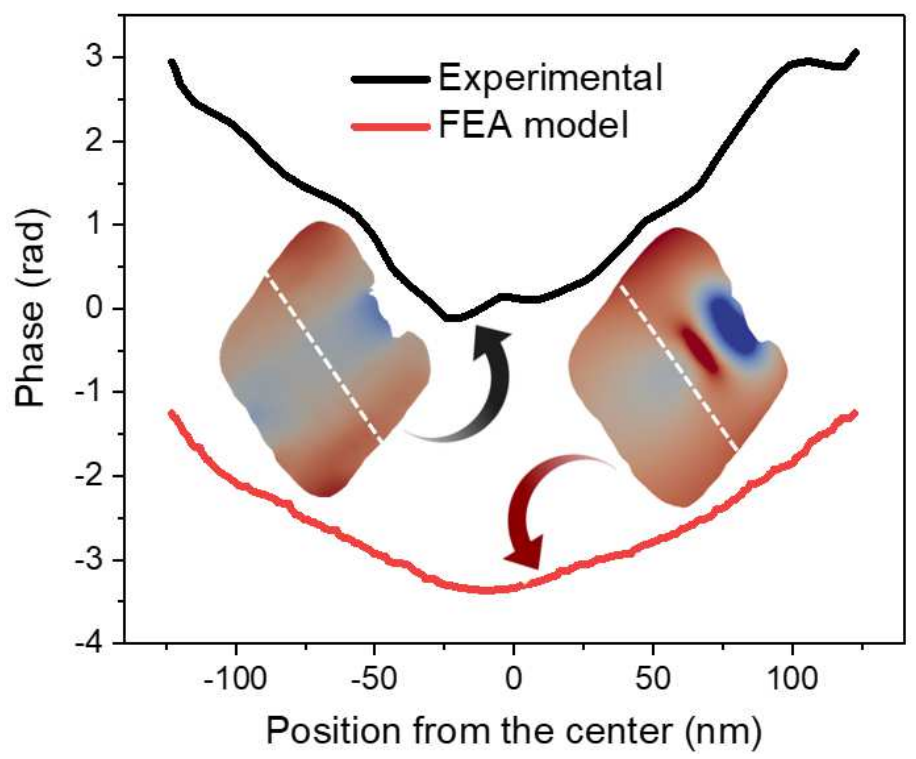

Figure 3. Phase line plot for the experimental data and the FEA model. The insets are cross-

533 sectional views of the experimental (middle-left) and FEA model (middle-right). The white dashed 534 lines represent the position of the phase line plot for the experimental result (solid black line) and 535 FEA model (solid red line).

536

(a)

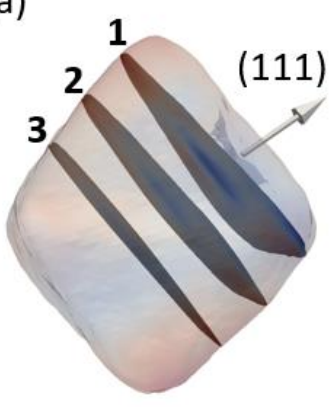

(b) 1
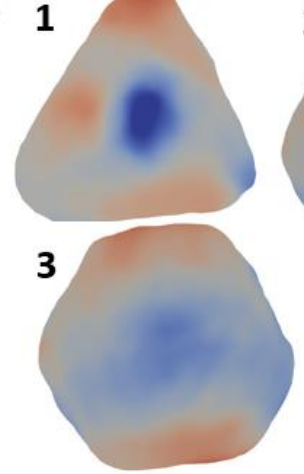
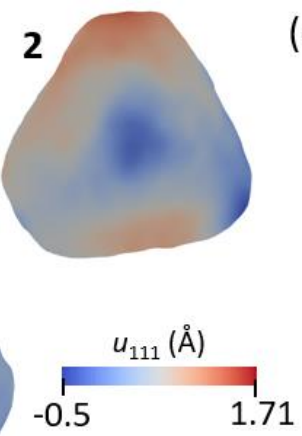

(c)
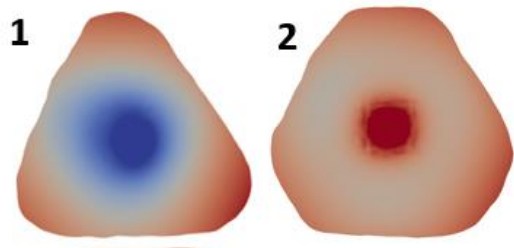

3

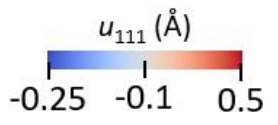

Figure 4. Cross-sectional views of the displacement distribution for the experimental data and the FEA model. (a) Spatial position of the cross-sections (1-3) shown in (b) and (c). The

541 nanocrystal is plotted as a semitransparent phase isosurface and the arrow represents the (111)

542 scattering vector. (b) Cross-sectional views for the experimental data and (c) the same slices for 543 the FEA model. 


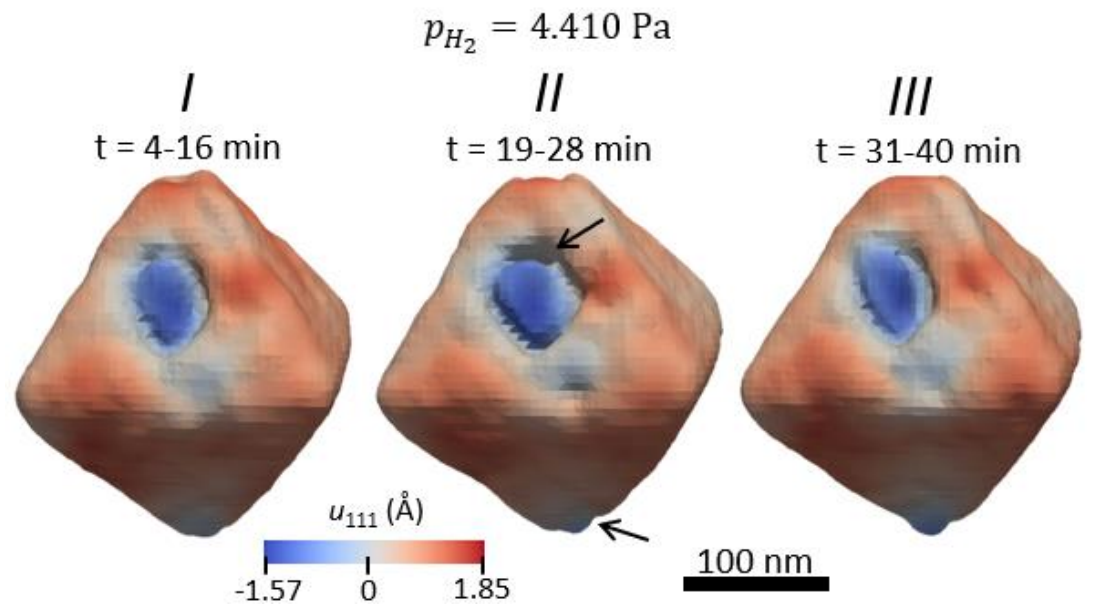

545 Figure 5. Displacement field evolution during the hydrogen exposure measurement $\left(\boldsymbol{p}_{\mathrm{H}_{2}}=\right.$

546 4. 410 Pa). Time evolution of the morphology and surface displacement distribution for the same

$547 \mathrm{Pd}$ nanoparticle measured under a hydrogen gas atmosphere. The hydrogen exposure time (t) is 548 indicated. 

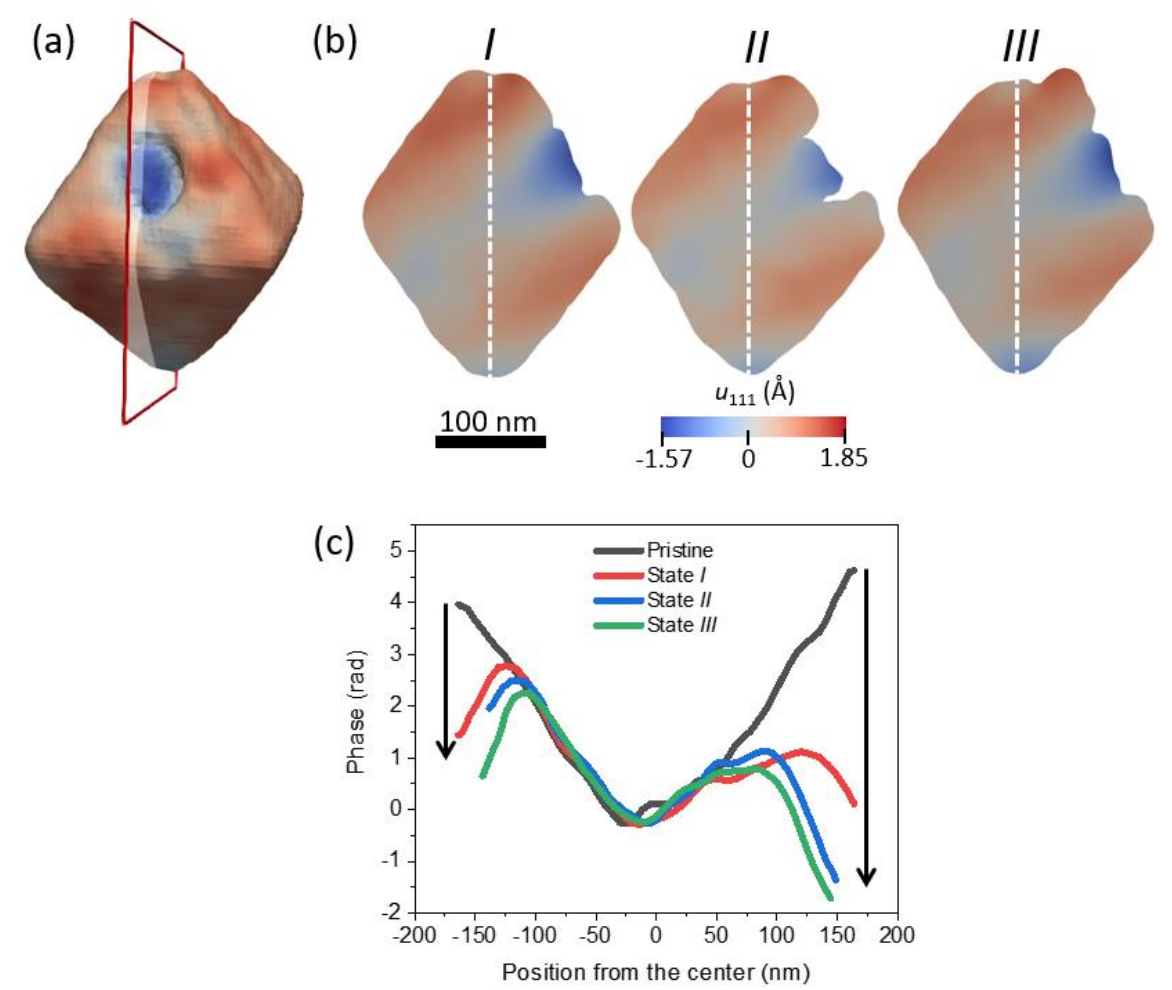

551 Figure 6. Cross-sectional views of the phase distribution for states $I$-III. (a) 3D reconstruction

552 with the transparent plane representing the position of the cross-sections shown in (b) for states $I$ 553 III. (c) Phase plot line over the dashed white line shown in (b) for states I-III. The black arrows at 554 the sides of the plot show the direction of the phase reversal. 


\section{Figures}
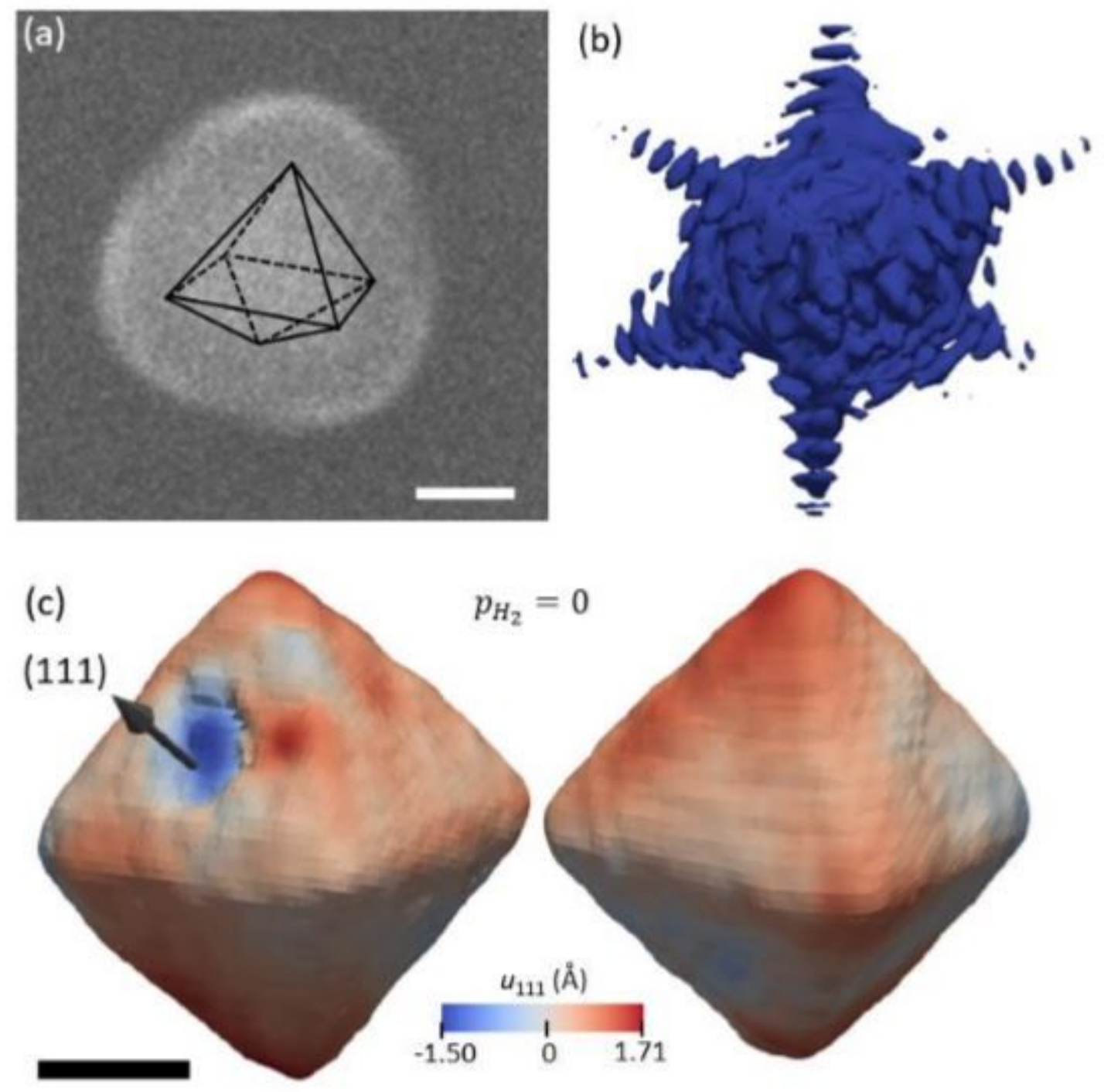

\section{Figure 1}

Imaging the displacement field of the octahedral Pd nanoparticle. (a) Scanning electron microscopy image of an individual Pd nanoparticle, showing the TiO2 layer surrounding the metallic core. The black dashed lines are a guide for the eyes, distinguishing the Pd surface and the titanium dioxide. (b) Isosurface of the 3D diffraction pattern obtained from the Pd nanoparticle measured under the pristine state $(\mathbb{\square} 2=0)$. (c) Different views of the reconstructed displacement field for the pristine state nanoparticle shown as an isosurface, colored according to its displacement component along the [111] direction, u111. The black arrow represents the [111] direction of the measurement Q-vector. The scale bars in (a) and (c) represent $100 \mathrm{~nm}$. 

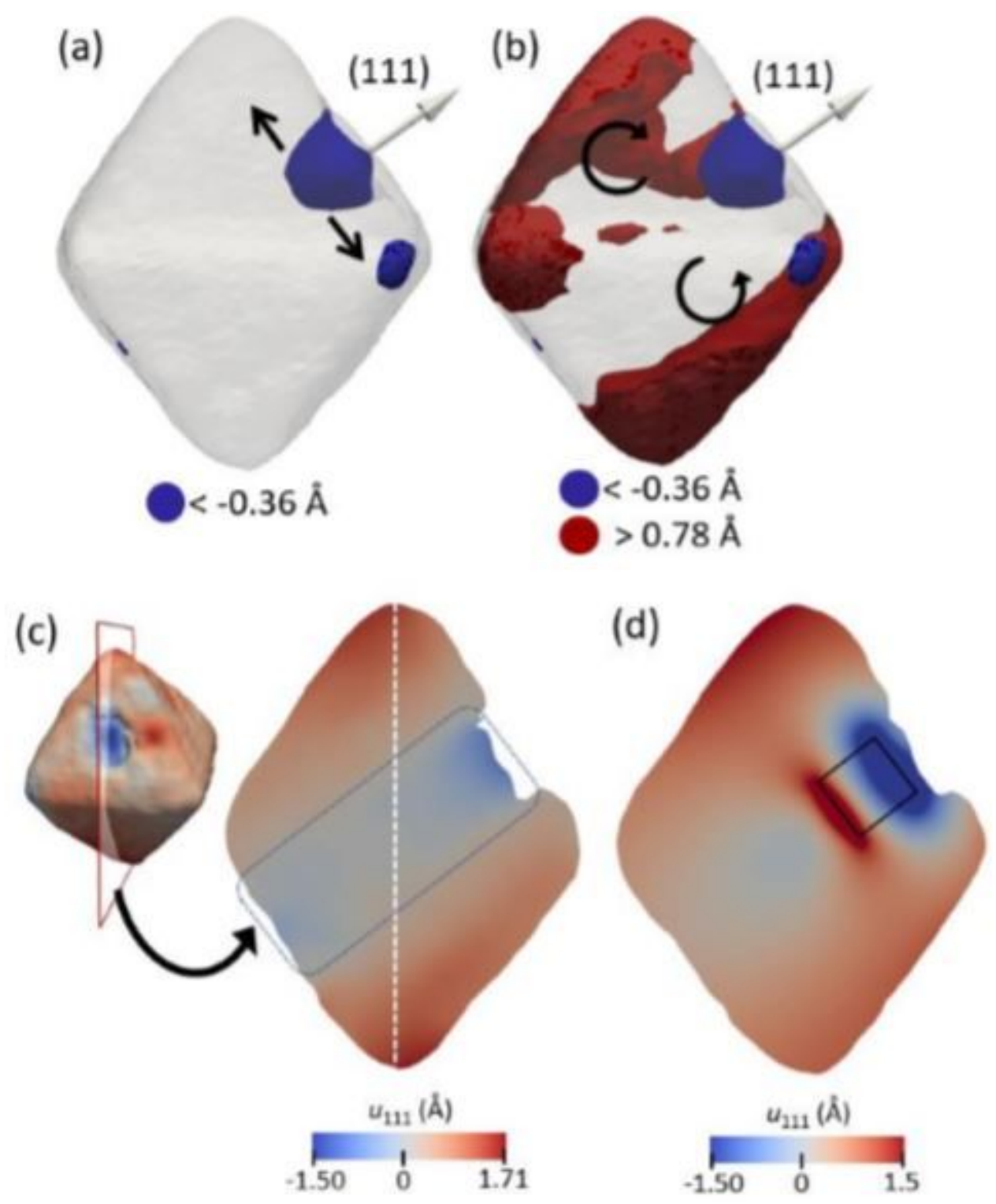

Figure 2

Displacement field distribution over the Pd nanocrystal for the measument done under pristine conditions. (a) Semitransparent 3D plot of the Pd nanoparticle showing negative displacement values (< $-0.36 \AA$, blue-colored) and (b) the same negative values shown in (a) and positive displacement values (> $0.78 \AA$, red-colored). (c) Cross-section view of the internal displacement field distribution taken along the (111) Q vector. The position of the plane taken from the 3D reconstruction is shown in the top-left. (d) Cross-sectional view of the displacement distribution from the finite element analysis, modeled using the original reconstructed octahedral nanoparticle shape with a cube placed inside it to represent the seed nanoparticle. The square inside the plot represents the cube position. 


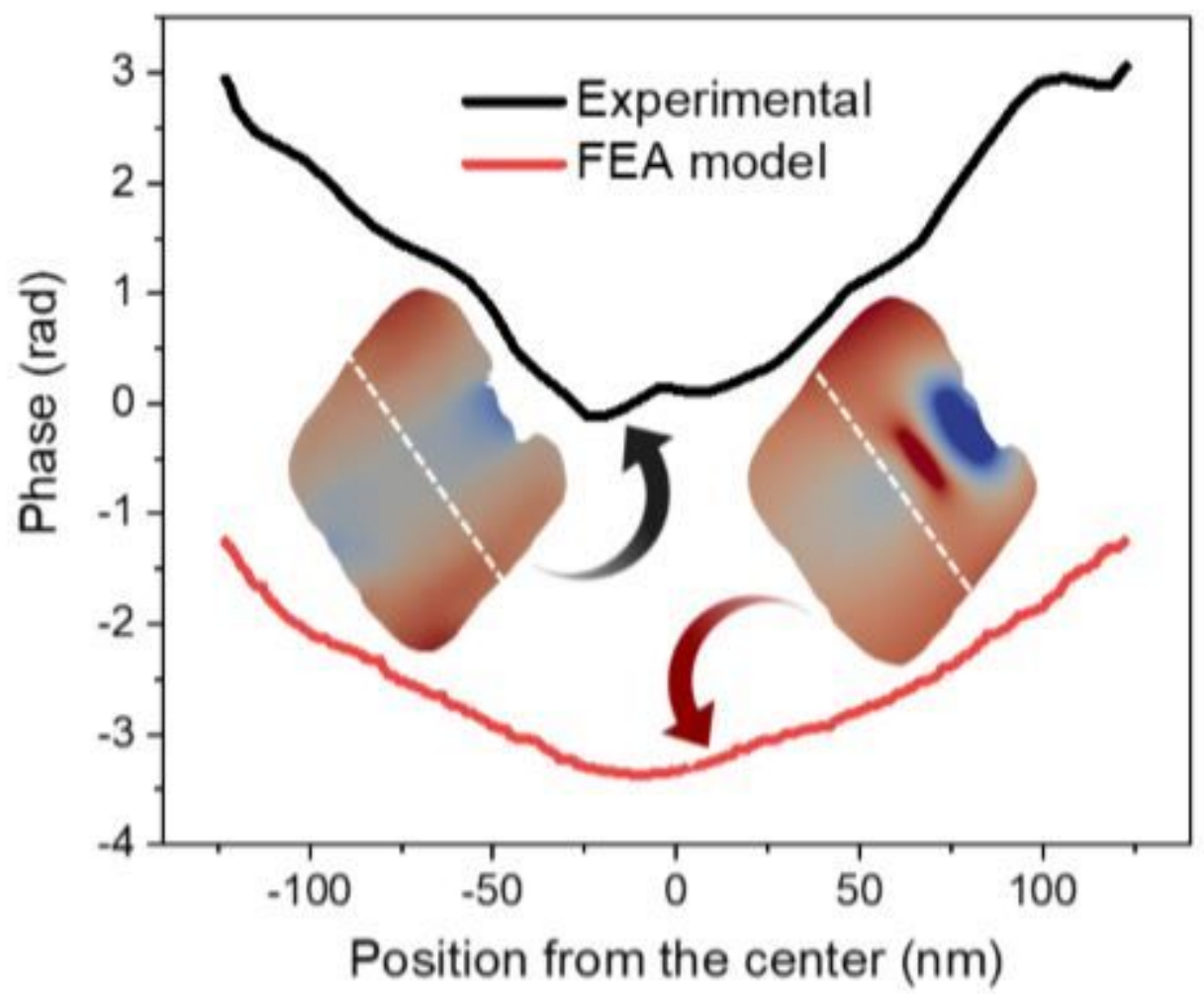

\section{Figure 3}

Phase line plot for the experimental data and the FEA model. The insets are cross- sectional views of the experimental (middle-left) and FEA model (middle-right). The white dashed lines represent the position of the phase line plot for the experimental result (solid black line) and FEA model (solid red line).

(a)

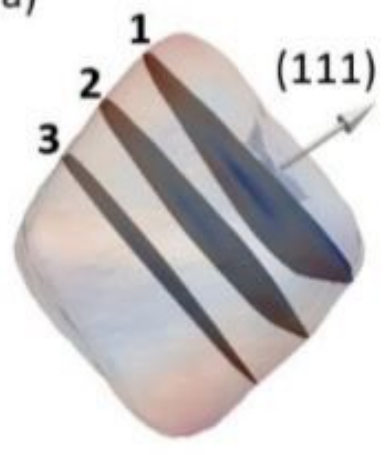

(b) 1

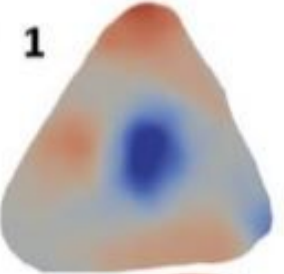

3
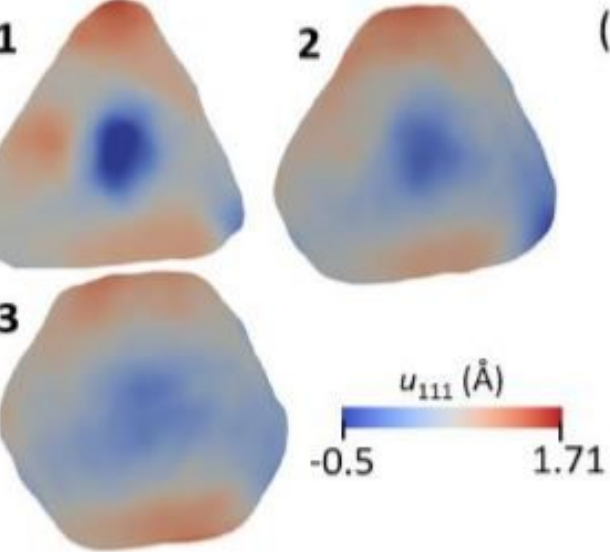

(c) 1
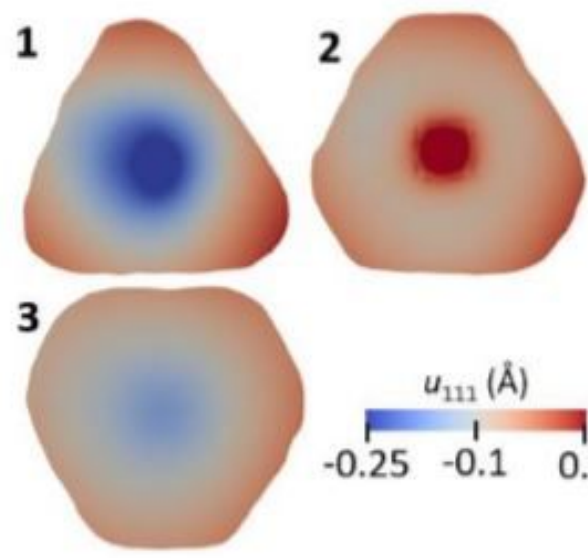

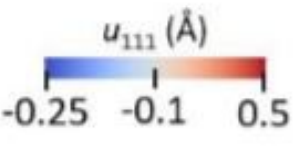

\section{Figure 4}

Cross-sectional views of the displacement distribution for the experimental data and the FEA model. (a) Spatial position of the cross-sections (1-3) shown in (b) and (c). The nanocrystal is plotted as a semitransparent phase isosurface and the arrow represents the (111) scattering vector. (b) Crosssectional views for the experimental data and (c) the same slices for the FEA model. 


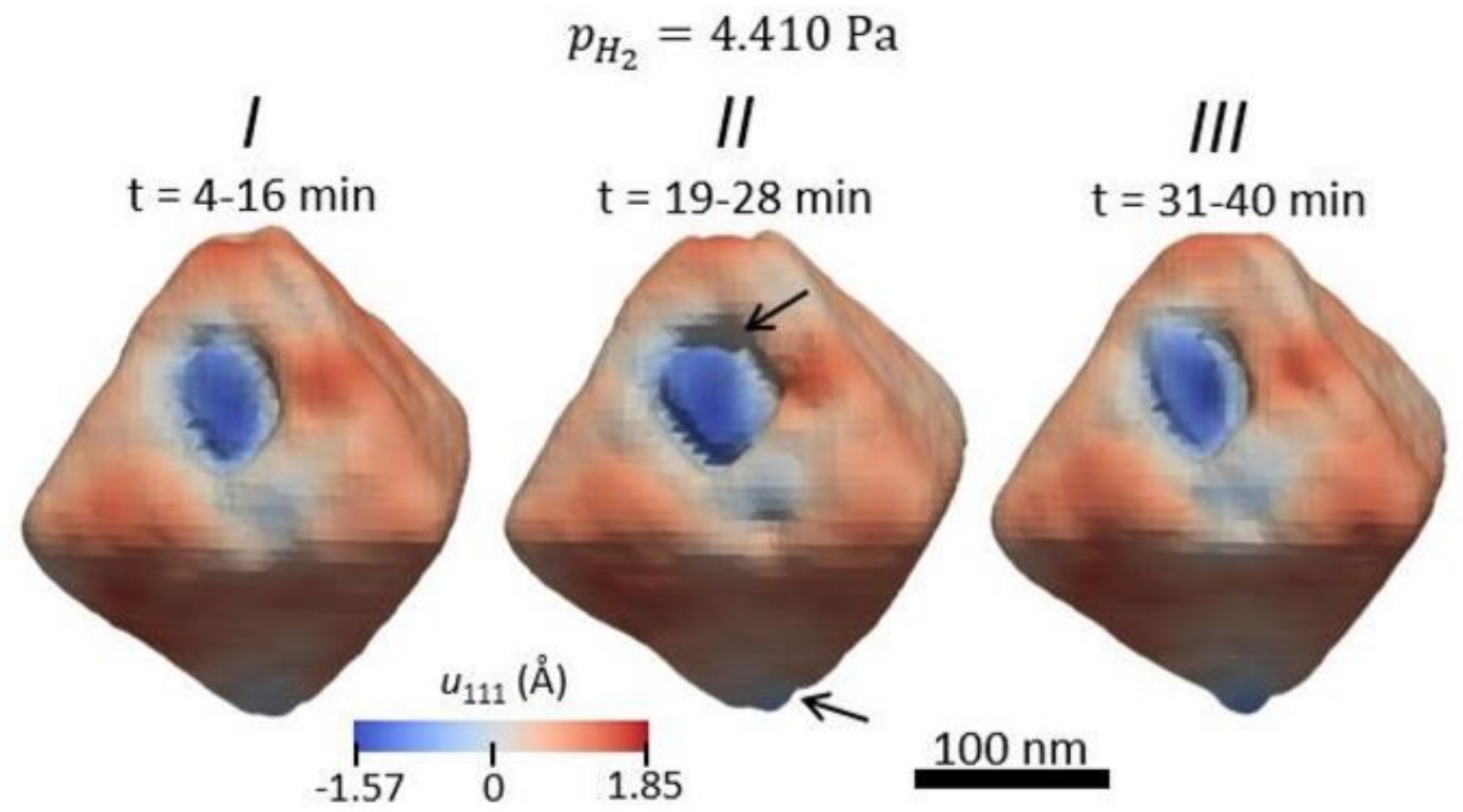

Figure 5

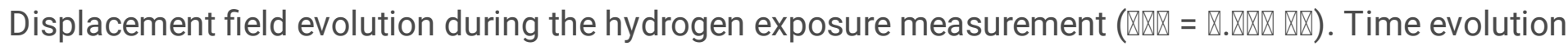
of the morphology and surface displacement distribution for the same Pd nanoparticle measured under a hydrogen gas atmosphere. The hydrogen exposure time $(t)$ is indicated.

\section{Supplementary Files}

This is a list of supplementary files associated with this preprint. Click to download.

- AFSPdhydrideSI.pdf 